\title{
INCREMENTO DE RENTAS EN ARGELIA, MARRUECOS Y TÚNEZ. ¿CÓMO AFECTA A LA SEGURIDAD ALIMENTARIA?
}

\author{
Antonio Alonso ${ }^{1}$ \\ Universidad San Pablo CEU / UNISCI
}

\begin{abstract}
Resumen:
El vecindario seguro que se pretende crear en la ribera sur del Mediterráneo pasa, también, por el desarrollo económico y político de los Argelia, Marruecos y Túnez. El autor señala cómo en estos tres países se ha dado un incremento de rentas de manera generalizada, tanto en el nivel macroeconómico como en el nivel de las rentas familiares, aunque de manera desigual, dadas las características de cada uno de estos países. No obstante, se puede afirmar que ese incremento generalizado de rentas conllevará una diversificación de la dieta y una mayor demanda de alimentos más caros.
\end{abstract}

Palabras clave: Magreb, Argelia, Marruecos, Túnez, seguridad alimentaria, incremento de rentas, diversificación en la dieta.

Title in English: "Rent Increase in Algeria, Morocco and Tunisia: Which are the Effecst on Food Security?"

\section{Abstract:}

In order to have a safe neighborhood at the Southern part of the Mediterranean it is necessary the economic and political development in Algeria, Morocco and Tunisia. The author explains how these countries have increased their incomes in a general way, at a macroeconomic level and at a household one, but with different results due to the characteristics of every country. However, it is possible to say that this generalized increase in income will lead to a diversification of diet and a demand for more expensive food.

Keywords: Maghreb, Algeria, Morocco, Tunisia, food security, increase of incomes, diversification of diet.

Copyright $\odot$ UNISCI, 2013.

Las opiniones expresadas en estos artículos son propias de sus autores, y no reflejan necesariamente la opinión de UNISCI. The views expressed in these articles are those of the authors, and do not necessarily reflect the views of UNISCI.

\footnotetext{
${ }^{1}$ El Dr. Antonio Alonso Marcos es profesor del Instituto de Humanidades "Ángel Ayala”, Universidad CEU-San Pablo, investigador senior de UNISCI y miembro del Foro Hispano-Argelino.

Dirección: Instituto de Humanidades “Ángel Ayala”, Universidad CEU San Pablo, Paseo Juan XXIII, 8, 28040, Madrid.

E-mail: aalonso@ceu.es.

http://dx.doi.org/10.5209/rev_UNIS.2013.n31.4470"
} 


\section{Introducción}

Cabe distinguir cuatro dimensiones básicas en la seguridad alimentaria: disponibilidad física de los alimentos; el acceso económico y físico a los alimentos; la utilización de los alimentos; y la estabilidad en el tiempo de las tres dimensiones anteriores. Parece evidente, pues, que es un concepto muy complejo y que se puede medir sólo a través del estudio cruzado de muchas variables tales como la tasa bruta de mortalidad, la prevalencia de malnutrición ${ }^{2}$, el acceso/disponibilidad de alimentos, la diversidad de la dieta, el acceso/disponibilidad de agua, las estrategias para enfrentar problemas o los activos para la subsistencia.

Estudiando el impacto del incremento de rentas o disminución de rentas en la seguridad alimentaria en los Estados del Magreb central, el foco de atención se pone en la demanda, en el acceso económico y físico de los alimentos y en si éstos son suficientes, están en buen estado y el aporte calórico que realizan a la dieta de los habitantes de esta región en concreto. Un aspecto que habría que destacar, además, en este campo, es si hay disponibilidad de dichos alimentos en el lugar o deben ser importados del exterior, ya que eso es un pilar fundamental de la seguridad alimentaria. Obviamente, un país será más seguro cuanto menos dependa de las importaciones, especialmente si se trata de productos tan básicos como son los alimentos.

La autosuficiencia en materia de alimentos es un capítulo esencial en la seguridad del Estado ya que, a diferencia de lo que sucede con otras materias primas, se puede prescindir de un elemento (oro, plata, azufre, hierro,...) y reorientar la producción industrial hacia otra actividad o cambiar de socios comerciales; ahí las posibilidades serían múltiples. Sin embargo, cuando lo que falta es el abastecimiento suficiente de alimentos y se depende en tan gran medida de las importaciones, eso afecta, irremediablemente, al juego internacional, donde, si se desea encontrar un equilibrio, se deberá plegar, desde una posición de desventaja, a los deseos de otros países. Parece que eso es claro con la dependencia energética, pero con el tema alimentario también sucede algo parecido.

Como afirmó el G-8 en el punto $\mathrm{n}^{\mathrm{o}} 4$ de la Declaración Conjunta de L'Aquila sobre Seguridad Alimentaria Global, "la seguridad alimentaria está conectada estrechamente con el crecimiento económico y el progreso social así como con la estabilidad económica y la paz". Además, acaba ese mismo apartado asegurando que "es necesario mejorar el acceso a la alimentación a través de una generación de ingresos y una distribución más equitativos, de la creación de empleo y de perspectivas de ingresos en los países en vías de desarrollo".

Por último, se hace necesario distinguir tres niveles de análisis a la hora de estudiar la seguridad alimentaria: el país, las familias y el individuo ${ }^{3}$. Como se puede deducir fácilmente, los datos macroeconómicos podrían llevarnos a conclusiones poco ajustadas a la realidad de la amplia mayoría de la población de dicho país, por lo que el nivel estatal quedaría descartado en este estudio. El nivel individual es por un lado muy difícil de medir y por otro es poco fiable.

\footnotetext{
2 "In addition, Pinstrup-Andersen and Shimokawa (2008) argue that in developing countries, health and nutritional status may be a better measure for deprivation than income per capita”, en: Maystadt, Jean-Francois; Trinh Tan, Jean-Francois y Breisinger, Clemens: "Does Food Security Matter for Transition in Arab Countries?", IFPRI Discussion Paper, no 1196 (Julio 2012), p. 4: ver también: Pinstrup-Andersen, P., y Shimokawa, S.: "Do Poverty and Poor Health and Nutrition Increase the Risk of Armed Conflict Onset?", Food Policy, vol. 6, no 33 (2008), pp. 513-520.

3 Ver: "Food Security: The Economic and Institutional Framework", en Implications of Economic Policy for Food Security: A Training Manual, IFPRI, en http://www.foodsecurityportal.org.
} 
El nivel familiar es el que mejor reflejaría la realidad social de un país ya que es lo suficientemente específico como para retratar la situación económica de un país y, además, no entraría al detalle de cuántas kilocalorías consume cada miembro de la familia, que para el propósito de este estudio no es un dato tan relevante. La familia, célula básica de la sociedad, es también la unidad básica a la hora de medir la economía real de un país. Además, en estos países de tradición árabe-islámica la familia no es un elemento más sino el pilar fundamental de la sociedad, por delante incluso de la nación o el Estado, conceptos occidentales que chocan con su "ummah". Tanto es así que su concepto de familia es un concepto mucho más extenso que el europeo de dos progenitores y uno o dos vástagos.

\section{La situación económica en esta región}

Para estudiar el incremento de rentas, el indicador más socorrido es el Producto Interior Bruto per capita (PIB pc) ya que muestra cuánta riqueza producida en un país le correspondería teóricamente - a cada habitante. No obstante, aunque ha habido un crecimiento prácticamente constante en el PIB desde el año 1999, es necesario fijarse en otros indicadores que retraten mejor la realidad social de estos países ya que puede ser que el país en sí haya incrementado su PIB gracias a los hidrocarburos o al aumento del turismo pero dicha riqueza no haya sido distribuida homogéneamente entre la población. Los porcentajes de crecimiento del PIB anual demuestran que el crecimiento en Argelia se ha dado de manera más homogénea (con cifras similares a las de un país desarrollado), en Marruecos ha sido más irregular, con periodos de crecimiento más espectaculares, similar al caso de Túnez, que se vio afectada por la crisis económica y financiera internacional y por los efectos negativos de la "primavera árabe" sobre el turismo:

Tabla 1. Crecimiento interanual del PIB (\%)

\begin{tabular}{|l|c|c|c|}
\hline & $\mathbf{1 9 9 9}$ & $\mathbf{2 0 0 8}$ & $\mathbf{2 0 1 1}$ \\
\hline Argelia & 3 & 2 & 2 \\
\hline Marruecos & 1 & 6 & 5 \\
\hline Túnez & 6 & 5 & -2 \\
\hline
\end{tabular}

Fuente: Banco Mundial

Los datos del PIB pc ponen de nuevo en evidencia que Marruecos es el menos desarrollado y que Argelia es el país con mayor riqueza. El PIB per capita ha evolucionado de la siguiente manera:

Tabla 2. PIB per capita (en US \$ actuales)

\begin{tabular}{|l|c|c|c|}
\hline & $\mathbf{1 9 9 9}$ & $\mathbf{2 0 0 8}$ & $\mathbf{2 0 1 1}$ \\
\hline Argelia & 1.616 & 4.967 & 5.244 \\
\hline Marruecos & 1.382 & 2.793 & 3.054 \\
\hline Túnez & 2.426 & 4.345 & 4.297 \\
\hline
\end{tabular}

Fuente: Banco Mundial 
Buceando entre estos datos macroeconómicos, el Banco Mundial afirmó que:

Los sectores de Argelia no relacionados con el petróleo y el gas siguieron creciendo en el ejercicio de 2010, impulsados por programas de inversión pública de gran envergadura financiados con los ingresos procedentes del sector de los hidrocarburos. El Banco volvió a tener participación en Argelia, donde contribuyó a abordar la diversificación económica; a diseñar, supervisar y evaluar el gasto público y las políticas sociales y económicas, y a reducir las disparidades espaciales ${ }^{4}$.

Con respecto a Marruecos, el Banco Mundial reconoce que este país ha hecho un esfuerzo notable para mejorar su situación incluso después de sobrevenida la crisis. El Banco Mundial también afirmó que dicha institución ha contribuido notablemente para que se diera esta mejora, incluso en el desarrollo rural $^{5}$.

El Producto Nacional Bruto (PNB) es un indicador parecido, ya que lo que revela es la cantidad de riqueza producida por los factores productivos de un país aunque lo hayan hecho en el extranjero y excluye a los factores de producción extranjeros que han generado riqueza dentro de las fronteras nacionales. Si se toma como referencia el inicio del siglo XXI, se puede afirmar que el PNB per capita de estos países disfrutó de un crecimiento sostenido:

Tabla 3. PNB per capita (en US \$ actuales)

\begin{tabular}{|l|c|c|c|}
\hline & $\mathbf{1 9 9 9}$ & $\mathbf{2 0 0 8}$ & $\mathbf{2 0 1 1}$ \\
\hline Argelia & 4.990 & 7.940 & 8.370 \\
\hline Marruecos & 2.460 & 4.240 & 4.910 \\
\hline Túnez & 4.920 & 8.390 & 9.090 \\
\hline
\end{tabular}

Fuente: Banco Mundial

Aquí se puede ver que el desarrollo económico experimentado por Túnez es mayor que el de Argelia y el de ésta que el de Marruecos, siendo este último país vecino más incómodo o el que supone un lastre para el desarrollo de la región. Que Túnez tenga tal desarrollo se explica en buena medida por las condiciones geográficas del país que, al ser relativamente pequeño y con una población proporcionada, le hace más gobernable y manejable. Además, los vecinos de Argelia y Marruecos -léase, especialmente, el Sahel- suponen factores de inestabilidad y amenazas a la seguridad mucho más serias que no las planteadas por los vecinos de Túnez excepción hecha del conflicto de Libia, claro está-, lo que anima también al turismo y a la

\footnotetext{
${ }^{4}$ Ver Banco Mundial: "Informe Anual 2010", p. 28.

5 Ibid., p 29: "Sus sólidas políticas macroeconómicas y su paquete de estímulo específico permitieron a Marruecos afrontar la segunda serie de efectos de la crisis. Se está empleando un préstamo para políticas de desarrollo por \$200 millones para respaldar los esfuerzos del Gobierno por incrementar el acceso de hogares y pymes al financiamiento y, al mismo tiempo, asegurar la estabilidad del sistema financiero. Se han otorgado dos nuevos préstamos para políticas de desarrollo, uno de ellos por \$100 millones (para la administración pública) y el otro por $\$ 60$ millones (para el sector de la educación), que forman parte de un programa de reformas de largo plazo. Otros \$370 millones de financiamiento de la inversión se distribuyen entre proyectos de desarrollo rural y de aprovechamiento del agua".
} 
inversión extranjera en general, con lo que eso supone de captación de capital extranjero. No obstante, no se puede olvidar que la influencia de los islamistas se ha incrementado en los tres países debido a la acción de la denominada "primavera árabe" que, muy al contrario de lo que se esperaba -un viraje hacia la democracia liberal—el sistema político y social se ha orientado hacia un fortalecimiento del islamismo, que es la fuerza política y social que durante 30 años ha sido la auténtica oposición al régimen dictatorial en Túnez, Egipto, Libia y el resto de naciones afectadas por la "primavera árabe".

También se puede ver que existen diferencias no sólo en los resultados (pasados) de creación de riqueza sino también una diferencia en cuanto a los planes de futuro y la inversión en infraestructuras, que estimulan la creación de nueva riqueza y de trabajo:

In total, eight out of 15 Arab-TI countries cut their per capita infrastructure spending, including Iran, Djibouti, Egypt, Lebanon, Morocco, Syria, Turkey, and Kuwait. The most drastic cuts occurred in Morocco (20.3 percent) and Turkey (13 percent), whereas infrastructure spending sharply increased by more than 15 percent per capita per year in Algeria, Jordan, and Oman ${ }^{6}$.

El coeficiente de Gini $^{7}$, creado por el estadístico italiano Corrado Gini para medir la desigualdad en los ingresos dentro de un país, revela que la riqueza en estos tres Estados está relativamente repartida ${ }^{8}$, con los siguientes coeficientes: Argelia $(0,353)$, Túnez $(0,408)$ y Marruecos $(0,409)$. Ocupan, respectivamente, los puestos $n^{\circ} 58,96$ y 99 de la tabla, de un total de $160^{9}$.

Un indicador que puede aportar más información extra es, por ejemplo, el número de desempleados y la tasa de desempleo de estos países. Antes de acudir a los resultados, planteemos las hipótesis:

1.- Si se ha incrementado el PIB de manera sostenida y el desempleo ha decrecido significaría que se ha creado riqueza en el país de manera extendida y generalizada, abarcando a más población. En este caso, la demanda de alimentos se habría incrementado y diversificado hacia productos de mejor calidad, aunque deban ser importados.

2.- Si se ha incrementado el PIB de manera sostenida, pero el desempleo ha crecido significaría que la riqueza que se ha generado se habría quedado concentrada en pocas manos. En este escenario, no se habría producido una demanda tan grande de alimentos ni la diversificación de los mismos hacia productos nuevos de mayor calidad sino hacia los tradicionales de la zona.

Los datos de la tasa de desempleo muestran cómo éste ha ido menguando en los últimos años, a pesar de la crisis financiera internacional, y ha pasado de un paro superior al $25 \%$ de la población activa a una situación de prácticamente pleno empleo (situado entre el 5 y el $8 \%$ de paro). Es muy probable que en estas buenas cifras de paro tenga algo que ver la emigración de nacionales a Europa, principalmente España y Francia. Los datos son los que siguen:

\footnotetext{
${ }^{6}$ Ver Ibid., p. 25.

${ }^{7}$ El coeficiente de Gini mide en una escala de 0 a 1 la distribución de la riqueza entre los habitantes de un país, siendo 0 la óptima distribución equitativa y 1 la pésima. Ver: Maystadt, Trinh Tan, y Breisinger, op. cit, p.11.

8 "Inequality as measured by the Gini coefficient suggests that wealth is distributed fairly equally in all Arab+ countries, with Gini coefficients between 0.32 and 0.41 (Table 2.1) with the exception of Comoros". Ver: Maystadt, Trinh Tan, y Breisinger, op. cit, p. 5.

${ }^{9}$ Ver el Informe de Desarrollo Humano de la ONU del año 2005.
} 
Tabla 4. Tasa de desempleo (\% de la población activa)

\begin{tabular}{|l|c|c|c|}
\hline País & $\mathbf{1 9 9 9}$ & $\mathbf{2 0 0 8}$ & $\mathbf{2 0 1 1}$ \\
\hline Argelia & 30 & 12,5 & 10 \\
\hline Marruecos & 23 & 10 & 8,9 \\
\hline Túnez & 16,5 & 14,1 & 18 \\
\hline
\end{tabular}

Fuente: Banco Mundial

Podría deducirse, por tanto, que la riqueza económica ha sido distribuida entre la población, como norma general, de manera algo más equitativa, lo que hace pensar que la demanda de alimentos se habría incrementado y diversificado. Esto se verá en el punto siguiente. No obstante lo más llamativo es la disparidad en la pobreza existente entre las zonas rurales y las ciudades en estos países.

Tabla 5. Población pobre (\%)

\begin{tabular}{|l|l|l|l|}
\hline País & $\begin{array}{l}\text { Porcentaje población } \\
\text { urbana pobre }\end{array}$ & $\begin{array}{l}\text { Porcentaje población } \\
\text { rural pobre }\end{array}$ & $\begin{array}{l}\text { Porcentaje de pobres en zonas } \\
\text { rurales }\end{array}$ \\
\hline Argelia & $10 \%$ & $15 \%$ & $52 \%$ \\
\hline Marruecos & $5 \%$ & $15 \%$ & $68 \%$ \\
\hline Túnez & $2 \%$ & $8 \%$ & $75 \%$ \\
\hline
\end{tabular}

Fuente: Improving Food Security in Arab Countries, World Bank (2009), p.13.

Por último, habría que hacer referencia a la variación de rentas en las zonas rurales. Estos países tienen un buen porcentaje de población -en torno a un tercio del total - viviendo en zonas rurales: Marruecos (más del 40\%), Túnez (más del 30\%) y Argelia (casi el 30\%). Aunque la UE constató que los índices de pobreza cayeron, por ejemplo, en Argelia, la pobreza sigue afectando más al campo ${ }^{10}$ que a las ciudades: "Poverty incidence in rural areas is more pronounced than in cities" ". Además, el Banco Africano de Desarrollo también es consciente de que "Although urban poverty is on the rise, poverty in Africa is largely a rural phenomenon" ". Esta es una tendencia que se ha ido consolidando y que, dado el proceso de urbanización, no tiene expectativas de que se invierta o cambie radicalmente.

\footnotetext{
${ }^{10}$ Para ver la incidencia de la pobreza en las zonas rurales ver los estudios del International Fund for Agricultural Development (IFAD), en http://www.ifad.org y en http://www.ruralpovertyportal.org/region/home/tags/africa. Ver ele studio más general Improving Food Security in Arab Countries, World Bank (2009); Para el caso marroquí, ver Crépon, Bruno; Devoto, Florencia; Duflo, Esther y Parienté, William: "Impact of Microcredit in Rural Areas of Morocco: Evidence from a Randomized Evaluation”, Agence Française de Développement, Impact Analyses Series, no 7 (Octubre de 2011).

${ }^{11}$ Ver Comisión Europea: Algeria Strategy Paper 2007 - 2013 \& National Indicative Programme 2007 - 2010, pp. 10-11.

${ }^{12}$ Ver African Development Bank African Development Fund: Bank Group Policy on Poverty Reduction, Febrero de 2004, pp. 5-6.
} 


\section{Cambios en la dieta debidos al incremento de rentas}

El incremento de rentas ha tenido una serie de efectos entre la población, específicamente en sus hábitos de consumo. En cuanto a la importación de alimentos, Argelia gasta un 3,5\% de su PIB con tal fin, Marruecos un 3,6\% y Túnez un 3,8\%, constituyendo un 16\%, un $11 \%$ y un $9 \%$ respectivamente del total de productos importados ${ }^{13}$, aunque en 2011 ascendieron hasta un $25 \%$ en el caso de Argelia. Este último caso es uno de los más inquietantes pues "Argelia se encuentra entre las zonas del mundo más dependientes de la importación de alimentos para satisfacer las necesidades de la población y se estima que esta dependencia puede seguir aumentando en el futuro" $" 14$.

En cuanto a la variedad ${ }^{15}$ de alimentos, se partiría de la premisa establecida por la FAO en su informe de 2012 titulado Hacia el futuro que queremos:

El futuro crecimiento de la demanda de alimentos estará determinado por las decisiones que adopten millones de consumidores a medida que crezcan las economías y se urbanice la población del planeta. La alimentación de la población pobre, incluso cuando es rica en hidratos de carbono, se caracteriza por la escasa variedad, diversidad y contenido de nutrientes, y suele carecer de suficientes micronutrientes. La reducción de la escasez de ingresos generará un volumen de gasto adicional considerable, así como nutricionalmente deseable, en alimentos, especialmente en proteínas, azúcares y grasas. La población de los países de ingresos altos (y, cada vez más, de los países de ingresos medianos) suele consumir más carne y grasas saturadas (así como azúcar y sal), pero a menudo no consume un volumen adecuado de fruta, verduras y granos enteros ${ }^{16}$.

El consumo de grasas y proteínas también es un elemento que influye, por tanto, en la seguridad alimentaria. A modo orientativo, en España se consumieron en 2009 alrededor de $150 \mathrm{~g}$ de grasa por persona y día y poco más de $100 \mathrm{~g}$ de proteínas por persona y día ${ }^{17}$. Ese mismo año, en Argelia se consumieron $90 \mathrm{~g}$ de proteínas por persona y día y alrededor de 65 g de grasa por persona y día; en Marruecos, $85 \mathrm{~g}$ de proteínas y $65 \mathrm{~g}$ de grasa por persona y día; y en Túnez, poco más de 80 g de grasa y 95 g de proteínas por persona y día. En los tres países de referencia, la evolución ha sido constantemente in crescendo, salvo el caso de Túnez, que ha sufrido distintas alteraciones a lo largo de la primera década del siglo XXI.

El incremento de rentas ha hecho que se importen alimentos ricos en grasas y proteínas, tales como cereales, aceite para cocinar, azúcar y lácteos. Sin embargo, el IFPRI califica a

\footnotetext{
${ }^{13}$ Fuente: Banco Mundial, 2009.

14 Ver González Torres, Francisco y Rojo Hernández, Carlos(2012): "Seguridad alimentaria en Argelia: Indicadores macroeconómicos. Producción agrícola y restricciones. Concepto de seguridad alimentaria y vulnerabilidad a la inseguridad alimentaria”, en Marquina, Antonio (ed.): Las relaciones hispano -argelinas: Contexto histórico, desafíos y proyectos comunes, Madrid, Foro Hispano Argelino, p. 166.

15 Ver García Álvarez-Coque, José María y López-García Usach, Teresa: "Los cambios en el consumo alimentario: repercusión en los productos mediterráneos", en Lamo de Espinosa, Jaime (coord.): El nuevo sistema agroalimentario en una crisis global, col. Mediterráneo Económico, no 15 (Mayo 2009), p. 248 y también p. 249.

${ }^{16}$ Ver FAO (2012): Hacia el futuro que queremos: Erradicación del hambre y transición a sistemas agrícolas y alimentarios sostenibles, Roma, p. 7.

${ }^{17}$ Fuente: FAOSTAT.
} 
Argelia y Marruecos como países con serio riesgo de inseguridad alimentaria ${ }^{18}$ y a Túnez le asigna un riesgo moderado ${ }^{19}$ :

Supporting growth that strengthens macro-level food security is particularly important for those countries with high macro-level vulnerability, such as Algeria, Egypt, Jordan, Lebanon, Sudan, Syria, Yemen, Comoros, Djibouti, Mauritania, Morocco, and the West Bank and Gaza ${ }^{20}$.

Por el contrario, FAOSTAT asignó para 2009 un consumo de poco más de 3.200 Kcal por persona y día a Argelia y Marruecos y poco más de $3.300 \mathrm{Kcal}$ a Túnez, cifra similar a la de España o Alemania aunque ligeramente inferior a las de Italia (3.480), Francia (3.550) o Portugal (3.650).

Esta disparidad de resultados podría justificarse porque se toman otros indicadores que reflejan que la realidad social está deteriorada, como por ejemplo la tasa de mortalidad infantil (por debajo de 5 años) o la del retraso del crecimiento en la población infantil ${ }^{21}$. Siguiendo a Breisinger, Ecker, Al-Riffai y Yu, estos son indicadores que relacionan la alimentación con el estado de salud y con el poder adquisitivo y ambos indicadores arrojan cifras muy elevadas para ser 2011, proyectando una realidad social poco alentadora, especialmente en Marruecos, como viene siendo norma general:

Tabla 6. Indicadores de impacto de la pobreza en la infancia (I)

\begin{tabular}{|l|c|cc|}
\hline País & Tasa de mortalidad infantil & $\begin{array}{l}\text { Prevalencia del retraso en el } \\
\text { crecimiento }\end{array}$ \\
\hline Argelia & 32,3 & 15,6 \\
\hline Marruecos & 37,5 & 21,6 \\
\hline Túnez & 20,7 & 9 \\
\hline
\end{tabular}

Fuente: Maystadt, Jean-Francois; Trinh Tan, Jean-Francois y Breisinger, Clemens: "Does Food Security Matter for Transition in Arab Countries?", IFPRI Discussion Paper, no 1196 (Julio de 2012), p. 3 .

\footnotetext{
18 "The index classifies countries into five groups based on their level of food insecurity risk. Bahrain, Kuwait, Oman, Qatar, Saudi Arabia, and UAE face low levels of food-insecurity risk. Algeria, Iran, Libya, Turkey, and Tunisia exhibit moderate food-insecurity risk, whereas all other countries experience serious, alarming, or extremely alarming levels of food insecurity risk. Among the countries with the most food-security risk are Somalia and Yemen, followed by Mauritania and Sudan”: ver Breisinger, Clemens; Ecker, Olivier; Al-Riffai, Perrihan y Yu, Bingxin (2012): Beyond the Arab Awakening: Policies and Investments for Poverty Reduction and Food Security, Washington DC, International Food Policy Research Institute, p. 28.

${ }^{19}$ Ibid., p. 34.

${ }^{20}$ Ibid., p. 31.

21 'Income-based measurements to assess progress in improving poor people's living conditions may be particularly optimistic. To check on this often-voiced concern, we first explore GDP per capita growth's relationships to both poverty and child under-nutrition (measured by child stunting) across Arab-TI countries and countries worldwide (and over time). (The prevalence of child undernutrition is a good indicator of general living conditions and poverty that is not income based)": Ibid., p. 7.
} 
Al hilo de estos datos, también se puede acudir a la tasa de malnutrición infantil, desagregando los datos provenientes del ámbito rural que, como se observa, son peores que en las ciudades ${ }^{22}$ :

Tabla 7. Indicadores de impacto de la pobreza en la infancia (II)

\begin{tabular}{|c|c|c|c|l|l|l|l|l|}
\hline País & \multicolumn{9}{l}{ Malnutrición infantil } & \multicolumn{2}{l|}{$\begin{array}{l}\text { Crecimiento annual per capita } \\
(\%)\end{array}$} & \multicolumn{2}{l|}{ Arco de elasticidad } \\
\hline & $\begin{array}{l}\text { Prevalencia } \\
(\%)\end{array}$ & $\begin{array}{l}\text { Cambio } \\
\text { anual }\end{array}$ & PIB & AgVA & MaVA & PIB & AgVA & MaVA \\
\hline Argelia & 15,9 & $-0,5$ & 1,4 & $-1,5$ & $-4,1$ & $-1,49$ & 1,67 & 0,73 \\
\hline Rural & 17,2 & $-0,8$ & & & & $-1,83$ & 2,04 & 0,89 \\
\hline Marruecos & 23,1 & $-0,6$ & 2,2 & 2,3 & 1,4 & $-0,77$ & $-0,73$ & $-1,27$ \\
\hline Rural & 29,3 & $-0,5$ & & & & $-0,60$ & $-0,57$ & $-0,99$ \\
\hline Túnez & 9,0 & $-2,0$ & 3,9 & 3,4 & 2,9 & $-1,35$ & $-1,60$ & $-1,91$ \\
\hline Rural & 12,7 & $-2,7$ & & & & $-1,33$ & $-1,58$ & $-1,88$ \\
\hline
\end{tabular}

Fuente: Breisinger, Ecker, Al-Riffai y Yu, op. cit., p. 16.

La "primavera árabe" ha sacudido el norte de África y ha supuesto un elemento de inestabilidad para todo el Mediterráneo, también en el ámbito de la seguridad alimentaria, especialmente por su impacto en la falta de ingresos:

The non-income-related effects of conflicts are also substantial. One year of conflict may throw countries 5 to 10 years back in social outcome indicators such as life expectancy and immunization rates (ESCWA 2011). Given the elevated risk of conflict during transition and the related high costs, it is important to understand why conflicts may arise. The most commonly cited causes of conflict are related to socioeconomic factors, geography, and institutions ${ }^{23}$.

No se puede olvidar, además, que, de acuerdo con el ACNUR, en junio de 2011 había en Túnez más de 40.000 refugiados libios. La acogida de un total de 41.412 libios en Túnez llevó, según el World Food Programme, al peligro de la malnutrición ${ }^{24}$ a un lugar donde generalmente había habido cierto equilibrio alimentario. No obstante, la inmensa mayoría de refugiados libios se hospedaron en casa de benefactores tunecinos (alrededor de un 60\%) o en casas de personas con los que había previos lazos familiares o laborales (30\%); muy pocos se alojaron en casas alquiladas por ellos mismos $(10 \%)^{25}$. Los cambios introducidos en la dieta de los libios en Túnez también son interesantes: menos carne, más pasta, menos cuscús, menos vegetales y casi nada de fruta, menos leche y de peor calidad, y agua mineral en

\footnotetext{
${ }^{22}$ AgVA: Valor agrícola añadido; MaVA: Valor manufacturado añadido. Ver: Breisinger; Ecker; Al-Riffai y Yu, op. cit., p. 16.

${ }^{23}$ Ver Maystadt; Trinh Tan; y Breisinger: "Does Food Security Matter for Transition in Arab Countries?", IFPRI Discussion Paper, $\mathrm{n}^{\circ}$ 1196, Julio de 2012, p. 1.

${ }^{24}$ Ver Niazi, Asif y Musa, Mario: "Rapid Food Security Assessment of Libyan Refugees in Tunisia”, World Food Programme (Junio de 2011), p. 8.

${ }^{25}$ Ibid., p. 6.
} 
condiciones poco higiénicas. Aunque eso no implicó malnutrición, sí trajo consigo numerosos casos de diarrea ${ }^{26}$.

\section{Conclusiones}

De manera general, se puede afirmar que de los tres países, Argelia es el Estado en el que se da un desarrollo económico sostenido en el tiempo y de manera algo más equitativa entre una buena parte de la población, Marruecos está menos equilibrado y el desarrollo llega sólo a ciertas capas de la población, y Túnez crece de manera más irregular, muy dependiente del turismo, pero con un reparto de riqueza más equitativo que el existente en Marruecos.

Los indicadores macroeconómicos revelan que en el nivel estatal, la economía ha mejorado, ha habido un incremento de rentas aunque en cada país con un ritmo distinto. Fruto de dicho incremento de rentas, también ha habido un aumento en los últimos diez años en la cantidad y variedad de alimentos, especialmente en aquellos ricos en grasas y proteínas. Dados los datos de crecimiento de población, urbanización y crecimiento de rentas de estos países es de esperar un incremento sostenido de su demanda de alimentos ${ }^{27}$.

Los indicadores económicos que se fijan en el nivel de la unidad familiar también muestran que ha habido un leve incremento de rentas, aunque persiste el riesgo de inseguridad alimentaria tal y como se percibe a través de otros indicadores como el de la prevalencia de la malnutrición infantil. Aun así, los datos parecen confirmar la tendencia en la dirección de que se está incrementando la demanda de alimentos y se está virando hacia una dieta más parecida a la de países desarrollados (menos vegetales, más grasas y proteínas), especialmente en las ciudades.

Como afirma la FAO en su informe Hacia el futuro que queremos: "La reducción de la escasez de ingresos generará un volumen de gasto adicional considerable, así como nutricionalmente deseable, en alimentos, especialmente en proteínas, azúcares y grasas"28. Esa dinámica, que se da de manera generalizada, se da de manera mucho más clara en los países que están haciendo una transición económica hacia el desarrollo pleno, con especial mención a los países de la zona mediterránea:

Los desarrollos más dinámicos se han producido en los países emergentes, especialmente en China e India, en donde el incremento de renta conlleva el consumo de alimentos de mayor valor añadido, especialmente de carnes y productos lácteos. Los cambios se están sucediendo también en la región mediterránea, con una pérdida progresiva de los hábitos que se asociaban a una dieta saludable. Sin duda, la caída del consumo de frutas y hortalizas frescas puede contarse como una de las causas de la crisis que atraviesa el sector en España, que debe innovar para adaptarse a los cambios en las preferencias de los consumidores ${ }^{29}$.

\footnotetext{
${ }^{26}$ Ibid., p. 8.

${ }^{27}$ Véase también World Bank 2009: Improving Food Security in Arab countries, pp. 17-29.

${ }^{28}$ Ver "Hacia el futuro que queremos...", p. 7.

${ }^{29}$ García Álvarez-Coque y López-García Usach, op. cit., p. 261.
} 